\title{
Changes of serum uric acid and its clinical correlation in children with dilated cardiomyopathy
}

\author{
Ting-Ting Li, Hao-Ying Li, Ji Cheng \\ Department of Cardiology, Tianjin Children's Hospital/Tianjin University Children's Hospital, Tianjin, China \\ Contributions: (I) Conception and design: TT Li; (II) Administrative support: J Cheng; (III) Provision of study materials or patients: TT Li, HY Li; \\ (IV) Collection and assembly of data: TT Li; (V) Data analysis and interpretation: TT Li; (VI) Manuscript writing: All authors; (VII) Final approval \\ of manuscript: All authors. \\ Correspondence to: Ting-Ting Li. Department of Cardiology, Tianjin Children's Hospital/Tianjin University Children's Hospital, Tianjin 300074, \\ China. Email: litingting201706@163.com.
}

Background: Dilated cardiomyopathy (DCM) is the most common type of childhood cardiomyopathy and uric acid (UA) is considered closely associated with cardiovascular disease. There are few reports about the relationship between serum UA level and DCM in children, and the present study aimed to analyze the changes and clinical correlation of the two.

Methods: The clinical data of 49 children under 16 years old and who were hospitalized with DCM, and 44 healthy children who underwent physical examination in the same period at Tianjin Children's Hospital from June 2015 to November 2019 were analyzed retrospectively.

Results: The 49 children in the case group included 17 males and 32 females, aged from 2 to 172 months. The case group were divided into New York Heart Association (NYHA) functional class I $(n=2)$, class II $(n=17)$, class III $(n=11)$, and class IV $(n=19)$. The 44 healthy children selected as the control group included 20 males and 24 females aged from 2 to 161 months. The serum UA level was detected, and an ultrasonic cardiogram was conducted in each child. The serum UA level, left ventricular end-diastolic diameter (LVEDD), left ventricular end-systolic diameter (LVESD), and left atrial diameter (LAD) of the case group were higher than that of the control group, while the left ventricular ejection fraction (LVEF) and left ventricular fractional shortening (LVFS) were lower than that of the control group, and significant statistical differences were seen between the two groups $(\mathrm{P}<0.01)$. The serum UA level, LVEDD, LVESD, and LAD of NYHAIII-IV class patients were higher than that of the NYHAI-II class, but LVEF and LVFS were lower than that of the NYHA I-II class, and there were significant statistical differences between the two groups $(\mathrm{P}<0.01)$. Statistical correlations were seen between the serum UA level and NYHA functional class, LVEDD, LVESD, LAD, LVEF, and LVFS (rs=0.599, 0.567, 0.579, 0.475, -0.333, -0.341, respectively, $\mathrm{P}<0.05)$.

Conclusions: Elevated serum UA levels exist in children with DCM and correlate with NYHA functional class and ultrasonic values. Change in serum UA levels may be used as a biomarker reflecting the severity of DCM in children.

Keywords: Uric acid; dilated cardiomyopathy (DCM); heart failure; ultrasonic cardiogram; child

Submitted Sep 26, 2021. Accepted for publication Dec 14, 2021.

doi: $10.21037 / \mathrm{tp}-21-537$

View this article at: https://dx.doi.org/10.21037/tp-21-537 


\section{Introduction}

Cardiomyopathy is a disease targeting the myocardium and negatively affecting cardiac function (1). Of several types of cardiomyopathy in children, dilated cardiomyopathy (DCM) is the most common and is characterized by ventricular dilatation and systolic dysfunction in the absence of hypertension, valvular, congenital, or ischemic heart disease $(2,3)$. The pathological manifestation includes ventricular dilatation, myocardial degeneration and fibrosis. Physiologically, decreased ventricular contraction leads to decreased cardiac output and elevated end-diastolic pressure, which is transmitted to pulmonary edema and increased right heart pressure (2). The clinical presentation ranges from asymptomatic to acute decompensated heart failure and cardiogenic shock (4). The clinical manifestations of children with DCM are heart failure, arrhythmia, thromboembolism, and sudden death, and with a high mortality rate, most children die of advanced heart failure. In children with DCM, guideline-directed medical therapy includes diuretic agents, $\beta$-blockers, ACE inhibitors, and other medications as appropriate and highrisk patients can be effectively managed with implantable cardioverter-defibrillator. Mechanical assist devices and extracorporeal membrane oxygenation demonstrate their usefulness in severe acute heart failure from DCM, and heart transplantation is recommended for children with severe end-stage heart failure from DCM refractory to treatment (3). DCM can result in a large medical cost burden because of repeated hospitalizations and the potential need for transplantation (5).

Detailed understanding of the disease has grown in recent years, and a deep relationship between elevated levels of serum uric acid (UA) and cardiovascular disease in adults has become a research hotspot. However, there are few reports on the relationship between serum UA level and DCM in children.UA is the end-product of purine metabolism and is considered a powerful free-radical scavenger in human plasma for protecting endothelial cells against damage by exogenous oxidants (6). Elevated levels of serum UA have been identified as potential factors for gout, hypertension, abnormal glucose metabolism, and dyslipidemia (7). UA is also strongly associated with the incidence, severity, and poor prognosis of cardiovascular diseases such as coronary heart disease, heart failure, atrial fibrillation, mitral regurgitation, and pulmonary hypertension (8-10). Yazicioğlu et al. reported that the elevated serum UA levels were correlated with the severity of functional mitral regurgitation and echocardiographic volume indices in adults with DCM (11). At present, there is no firm consensus on the relationship between serum UA levels and DCM in children, and the present study aimed to analyze the changes and clinical correlation of serum UA in children with DCM.

We present the following article in accordance with the STROBE reporting checklist (available at https://dx.doi. org/10.21037/tp-21-537).

\section{Methods}

A total of 49 children with DCM less than 16-years-old who were hospitalized in Tianjin Children's Hospital from June 2015 to November 2019 were recruited as the case group, including 17 males and 32 females, aged from 2 to 172 months. All cases were diagnosed as DCM according to World Health Organization/International Society of Federation of Cardiology diagnostic criteria (12). Exclusion criteria were severe liver and kidney disease, hypertension, congenital heart disease, ischemic heart disease, valvular heart disease, arrhythmia, hemoglobin less than $10 \mathrm{~g} / \mathrm{dl}$, endocrine, and metabolic disorders (13). Children in the case group were divided into New York Heart Association (NYHA) functional class I $(\mathrm{n}=2)$, NYHA II $(\mathrm{n}=17)$, NYHA III ( $\mathrm{n}=11)$, and NYHA IV ( $\mathrm{n}=19)$. A control group composed of 44 healthy children including 20 males and 24 females aged from 2 to 161 months underwent physical examinations in the same period and were matched in age and gender with the case group. There was no significant difference in sex, age, and body weight between the two groups $(\mathrm{P}>0.05)$ (Table 1). All procedures performed in this study involving human participants were in accordance with the Declaration of Helsinki (as revised in 2013). The study was approved by Ethics and Research Committee of the Hospital (No. L2021-14). Individual consent for this retrospective analysis was waived.

Blood samples were obtained from each child in the fasting state, and the serum UA level was detected by a BECKMAN AU2700 automatic chemistry analyzer (Beckman Coulter, Brea, California, USA). Ultrasonic values including the left ventricular end diastolic diameter (LVEDD), left ventricular end systolic diameter (LVESD), left ventricular ejection fraction (LVEF), left ventricular fractional shortening (LVFS), and left atrial diameter (LAD)were observed by a Philips IE33 
Table 1 Characteristics of recruited children in the case and control group

\begin{tabular}{lcccc}
\hline Groups & Number & Males/females & Age (months) & Weight (kg) \\
\hline Case group & 49 & $17 / 32$ & $17.0(5.5-71.0)$ & $10.1(7.6-18.7)$ \\
Control group & 44 & $20 / 24$ & $33.1(8.0-99.0)$ & $14.4(8.4-30.4)$ \\
P value & & 0.290 & 0.378 & 0.210 \\
\hline
\end{tabular}

Table 2 Serum UA level and ultrasonic values of recruited children in the case group and control group

\begin{tabular}{|c|c|c|c|c|c|c|c|}
\hline Groups & Number & $\mathrm{UA}(\mu \mathrm{mol} / \mathrm{L})$ & LVEDD (mm) & LVESD (mm) & LVEF (\%) & LVFS (\%) & LAD (mm) \\
\hline Control group & 44 & $230.43 \pm 55.84$ & $30.95 \pm 7.78$ & $19.77 \pm 4.76$ & $68.70 \pm 4.61$ & $36.79 \pm 4.99$ & $17.66 \pm 4.22$ \\
\hline $\mathrm{t}$ value & & 6.949 & 7.638 & 10.737 & -19.66 & -16.65 & 4.89 \\
\hline $\mathrm{P}$ value & & 0.000 & 0.000 & 0.000 & 0.000 & 0.000 & 0.000 \\
\hline
\end{tabular}

UA, uric acid; LVEDD, left ventricular end-diastolic diameter; LVESD, left ventricular end-systolic diameter; LAD, left atrial diameter; LVEF, left ventricular ejection fraction; LVFS, left ventricular fractional shortening.

Table 3 Serum UA level and ultrasonic values of NYHA I-II and NYHA III-IV in the case group

\begin{tabular}{|c|c|c|c|c|c|c|c|}
\hline Groups & Number & $\mathrm{UA}(\mu \mathrm{mol} / \mathrm{L})$ & LVEDD (mm) & LVESD (mm) & LVEF (\%) & LVFS (\%) & LAD (mm) \\
\hline NYHA III-IV & 30 & $442.13 \pm 153.38$ & $51.43 \pm 14.28$ & $44.73 \pm 12.75$ & $26.55 \pm 8.97$ & $12.57 \pm 4.45$ & $26.9 \pm 8.99$ \\
\hline $\mathrm{t}$ value & & 3.862 & 3.134 & 3.360 & -3.128 & -3.16 & 3.394 \\
\hline$P$ value & & 0.000 & 0.003 & 0.002 & 0.004 & 0.001 & 0.001 \\
\hline
\end{tabular}

UA, uric acid; NYHA, New York Heart Association; LVEDD, left ventricular end-diastolic diameter; LVESD, left ventricular end-systolic diameter; LAD, left atrial diameter; LVEF, left ventricular ejection fraction; LVFS, left ventricular fractional shortening.

ultrasonic diagnostic instrument (Philips Medical System, Massachusetts, USA).

\section{Statistical analysis}

SPSS 16.0 (IBM Corporation, Armonk, NY, USA) was used for the statistical analysis. Continuous variables with normal distribution were expressed using means \pm standard deviations and the comparison between groups was detected using Student's $t$-test. Continuous variables with non-normal distribution were expressed using median (interquartile range) and the comparison between the groups was detected using the Mann-Whitney $U$ test in nonparametric test. Categorical variables were presented as percentages and the comparison between groups was detected using Chi-square test. Correlation analysis used Spearman rank correlation, and a $\mathrm{P}$ value of less than 0.05 was considered statistically significant.

\section{Results}

\section{Serum UA level and ultrasonic values between case group and control group}

Serum UA and ultrasonic values (LVEDD, LVESD, and LAD) of the case group were higher than that of the control group, while the LVEF and LVFS were lower than that of the control group. There were significant statistical differences between the two groups $(\mathrm{P}<0.01)$ (Table 2).

\section{Serum UA level and ultrasonic values between different NYHA functional classes in the case group}

In the case group, the serum UA level, LVEDD, LVESD, and LAD of the NYHA III-IV group were higher than that of NYHA I-II group, while the LVEF and LVFS were lower than that of the NYHA I-II group. There were significant statistical differences between the two groups $(\mathrm{P}<0.01)$ (Table 3). 


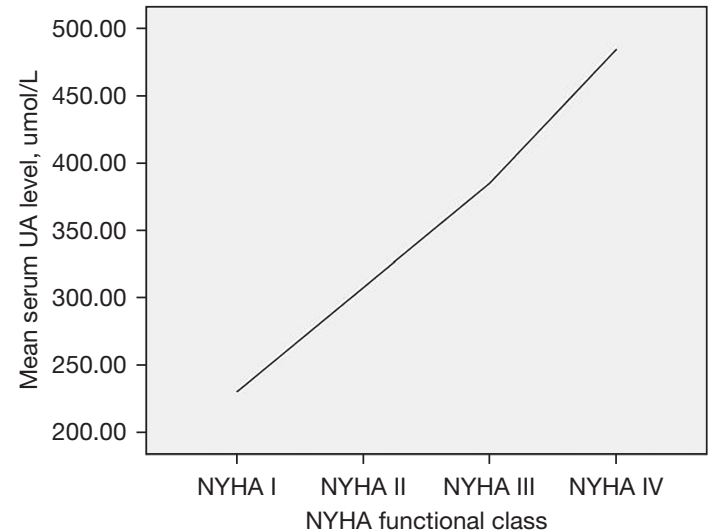

Figure 1 Correlation between serum UA level and NYHA functional class. UA, uric acid; NYHA, New York Heart Association.

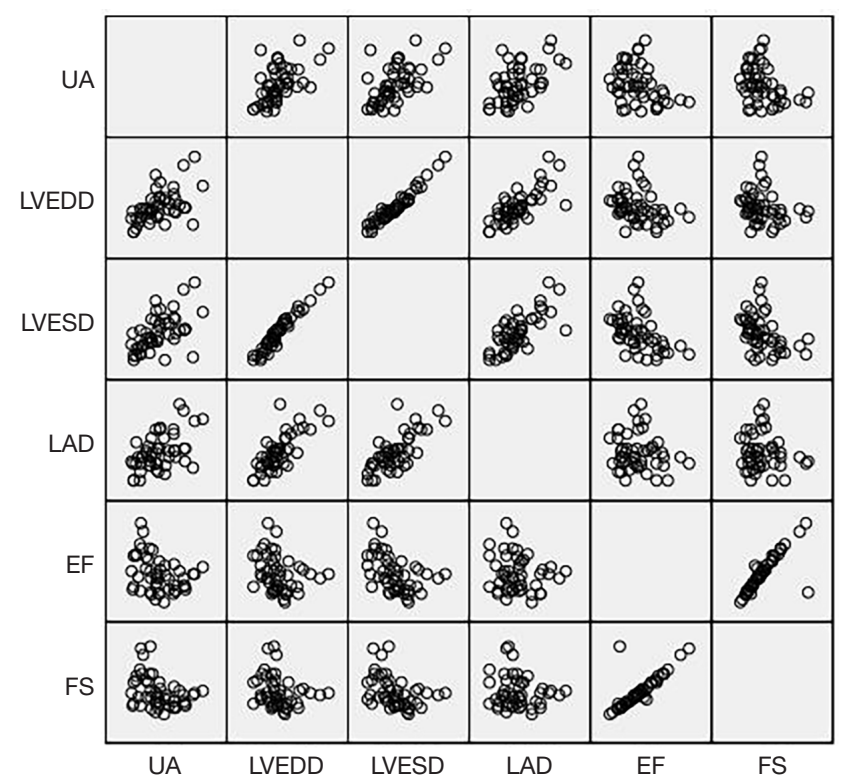

Figure 2 Correlation between serum UA level and LVEDD, LVESD, LAD, LVEF, and LVFS. UA, uric acid; LVEDD, left ventricular end-diastolic diameter; LVESD, left ventricular endsystolic diameter; LAD, left atrial diameter; LVEF, left ventricular ejection fraction; LVFS, left ventricular fractional shortening.

\section{Correlations between serum UA level and different variables}

The serum UA level correlated positively with the NYHA functional class ( $\mathrm{rs}=0.599, \mathrm{P}=0.000$ ) (Figure 1) and correlated positively with LVEDD ( $\mathrm{rs}=0.567, \mathrm{P}=0.000)$. The serum UA level also correlated positively with LVESD ( $\mathrm{rs}=0.579$,
$\mathrm{P}=0.000$ ) and correlated positively with $\mathrm{LAD}$ ( $\mathrm{rs}=0.475$, $\mathrm{P}=0.001)$. The serum UA level correlated negatively with LVEF ( $\mathrm{rs}=-0.333, \mathrm{P}=0.020$ ) and correlated negatively with LVFS ( $\mathrm{rs}=-0.341, \mathrm{P}=0.017$ ) (Figure 2).

\section{Discussion}

The overall prognosis of children with DCM is poor and the total rates of mortality and cardiac transplantation rate are high. In 2012, Pahl et al. reported the 5-year incidence of sudden cardiac death (SCD) to be $3 \%$ in a study containing 1,803 children who were considered the diagnosis of DCM (14). A national longitudinal cohort study in Australia in 2015 showed that the cumulative incidence of SCD at 15 years after diagnosis was 5\% (95\% CI: 2-11\%) in children with DCM (15), while Mets et al. reported the rates of mortality and cardiac transplantation for children with DCM to be as high as $20 \%$ and $25 \%$, respectively (16). DCM in children not only increases the social and family medical burden, but also seriously affects the physical and mental development of children and research to identify a biomarker of DCM has great significance for disease control and prognosis (17).

The relationship between serum UA levels and DCM in adults has attracted increasing attention in recent years. UA has attracted much attention as a parallel serum marker of oxidative stress, as in a hydrophobic environment, it loses its antioxidant ability and becomes a strong pro-oxidant in the presence of lipid peroxides (18), initiating cell damage and an inflammatory response. While hyperuricemia has been determined to be strongly associated with the occurrence, severity, and mortality of cardiovascular disease in adults, there are few reports about the relationship between serum UA level and DCM in children. Therefore, we conducted a retrospective case-control study of 49 children under 16 years old with DCM and 44 matched healthy children. We observed that compared with the control group, the serum UA level of the case group was higher, and that the level seen in the NYHAIII-IV group was higher than that of the NYHA I-II group $(\mathrm{P}<0.05)$. Our results suggest that elevated levels of serum UA exist in children with DCM and have a certain association with their cardiac function. However, whether the change of serum UA level was associated with the severity of DCM was not at first evident.

Accordingly, we further studied the case group data and presented a correlation analysis between serum UA level and different variables. Echocardiography aids in diagnosing DCM and determining the functional severity of the disease. 
Parameters derived from echocardiographic tissue imagingtissue Doppler, strain, strain rate, postsystolic shortening and others-are extensively used to provide a framework in evaluation and management of cardiomyopathies. A higher LVEDD combined with lower left ventricle function variables - most commonly assessed by LVEF and LVFSsuggests a dilated, hypokinetic phenotype which can be differentiated from restrictive phenotype by the presence of dilated atria and impaired myocardial compliance, and hypertrophic phenotype by the presence of greater wall thicknesses and pattern of thickening. Other feature includes mitral regurgitation, elevated pulmonary pressures, dilated right ventricle and impairment of diastolic function and so on. The reverse remodeling and improved LVEF could be seen while on medical therapy $(2,19)$. The results showed that serum UA level was correlated positively with NYHA functional class, correlated positively with LVEDD, LVESD, and LAD, and correlated negatively with LVEF and LVFS in the case group. This suggests there is a significant statistical correlation between change in serum UA level and the severity of DCM in children. High levels correlated with worsening heart disease and more severe ultrasonic values. The aggravation of DCM in children is accompanied by progressive myocardial remodeling and abnormal hemodynamics, which leads to continuous deterioration of hypoxic-ischemic states, followed by a progressive increase of serum UA levels. The related mechanisms are (I) ATP is degraded to adenine and xanthine, and there is an increased generation of xanthine oxidase. The increased availability of substrate (xanthine) and enzyme (xanthine oxidase) results in increased UA generation. (II) Release of lactic acid blocks urate secretion in the proximal tubules. (III) A low renal blood flow stimulates urate reabsorption (20). Elevated levels of serum UA have other negative consequences in heart failure, arrhythmia, pulmonary hypertension, thromboembolism, and myocardial remodeling by stimulating oxidative stress and inflammatory mechanisms, impairing endothelial function, activating the renninangiotensin system and circulating platelets, and mediating the proliferation of vascular smooth muscle cell, affecting endoplasmic reticulum calcium outflow and its consequences (21-25). These changes prompt the aggravation of DCM in children.

Our study revealed that elevated levels of serum UA exist in children with DCM and with the gradual deterioration of the condition, these levels increased progressively, and correlated with NYHA functional class and ultrasonic values. The elevated level of serum UA prompts cardiac damage in many ways, resulting in the deterioration of hemodynamics, decreasing systolic and diastolic function, and progressively aggravating heart failure, resulting in a poor prognosis. Our results demonstrate that serum UA levels might be used as a biomarker reflecting the severity of DCM in children and have high clinical value for formulating personalized treatment plants for pediatric cardiologists to reduce the social and family medical burden. Combining the results of our study with those of Kim et al. (25), paves the way for further study to determine whether serum UA can be used as a prognostic marker in children with DCM.

This study has some limitations. Firstly, the serum UA level may have been affected by some unmeasured confounders, such as the use and dose of diuretics $(26,27)$, respiratory status, family history, and life style. There are also conflicting results on whether diuretics affect the cardiovascular damage caused by elevated serum UA (27-31). Secondly, this was a single-center study using a small sample size, and we look forward to large sample multicenter studies to confirm our results. Thirdly, this study is a retrospective analysis, which is likely to cause some deviations in the results. It needs to be further confirmed by multi-center clinical trials.

\section{Acknowledgments}

Funding: None.

\section{Footnote}

Reporting Checklist: The authors have completed the STROBE reporting checklist. Available at https://dx.doi. org/10.21037/tp-21-537

Data Sharing Statement: Available at https://dx.doi. org/10.21037/tp-21-537

Conflicts of Interest: All authors have completed the ICMJE uniform disclosure form (available at https://dx.doi. org/10.21037/tp-21-537). The authors have no conflicts of interest to declare.

Ethical Statement: The authors are accountable for all aspects of the work in ensuring that questions related to the accuracy or integrity of any part of the work are appropriately investigated and resolved. All procedures performed in this study involving human participants were in accordance with the Declaration of Helsinki (as revised 
in 2013). The study was approved by Ethics and Research Committee of the Hospital (No.: L2021-14). Individual consent for this retrospective analysis was waived.

Open Access Statement: This is an Open Access article distributed in accordance with the Creative Commons Attribution-NonCommercial-NoDerivs 4.0 International License (CC BY-NC-ND 4.0), which permits the noncommercial replication and distribution of the article with the strict proviso that no changes or edits are made and the original work is properly cited (including links to both the formal publication through the relevant DOI and the license). See: https://creativecommons.org/licenses/by-nc-nd/4.0/.

\section{References}

1. Dadson K, Hauck L, Billia F. Molecular mechanisms in cardiomyopathy. Clin Sci (Lond) 2017;131:1375-92.

2. Lipshultz SE, Law YM, Asante-Korang A, et al. Cardiomyopathy in Children: Classification and Diagnosis: A Scientific Statement From the American Heart Association. Circulation 2019;140:e9-e68.

3. Lee HJ, Kim HK, Kim M, et al. Clinical impact of atrial fibrillation in a nationwide cohort of hypertrophic cardiomyopathy patients. Ann Transl Med 2020;8:1386.

4. Lee TM, Hsu DT, Kantor P, et al. Pediatric Cardiomyopathies. Circ Res 2017;121:855-73.

5. Park HY. Hereditary Dilated Cardiomyopathy: Recent Advances in Genetic Diagnostics. Korean Circ J 2017;47:291-8.

6. Puddu P, Puddu GM, Cravero E, et al. Relationships among hyperuricemia, endothelial dysfunction and cardiovascular disease: molecular mechanisms and clinical implications. J Cardiol 2012;59:235-42.

7. Zhi L, Yuzhang Z, Tianliang H, et al. High Uric Acid Induces Insulin Resistance in Cardiomyocytes In Vitro and In Vivo. PLoS One 2016;11:e0147737.

8. Lee SJ, Oh BK, Sung KC. Uric acid and cardiometabolic diseases. Clin Hypertens 2020;26:13.

9. Chinese Expert Consensus Group on diagnosis and treatment of Cardiovascular Disease combined asyptomatic hyperuricemia. The diagnosis and treatment advice of cardiovascular disease combined asymptomatic hyperuricemia (second edition). Chinese Journal of Cardiovascular Research 2012;10:241-9.

10. Turker Y, Ekinozu I, Turker Y, et al. High levels of highsensitivity C-reactive protein and uric acid can predict disease severity in patients with mitral regurgitation. Rev
Port Cardiol 2014;33:699-706.

11. Yazicioğlu MV, Avci A, Açar G, et al. Elevated uric acid and functional mitral regurgitation in dilated cardiomyopathy. Eur Rev Med Pharmacol Sci 2012;16:1637-41.

12. Maron BJ, Towbin JA, Thiene G, et al. Contemporary definitions and classification of the cardiomyopathies: an American Heart Association Scientific Statement from the Council on Clinical Cardiology, Heart Failure and Transplantation Committee; Quality of Care and Outcomes Research and Functional Genomics and Translational Biology Interdisciplinary Working Groups; and Council on Epidemiology and Prevention. Circulation 2006;113:1807-16.

13. Noori NM, Mahjoubifard M, Shahramian I, et al. Comparison between procalcitonin, brain natriuretic peptide, and uric acid in children with cardiomyopathy and controls. Biomed Res Int 2015;2015:510450.

14. Pahl E, Sleeper LA, Canter CE, et al. Incidence of and risk factors for sudden cardiac death in children with dilated cardiomyopathy: a report from the Pediatric Cardiomyopathy Registry. J Am Coll Cardiol 2012;59:607-15.

15. Bharucha T, Lee KJ, Daubeney PE, et al. Sudden death in childhood cardiomyopathy: results from a long-term national population-based study. J Am Coll Cardiol 2015;65:2302-10.

16. Mets G, Panzer J, De Wolf D, et al. An Alternative Strategy for Bridge-to-Transplant/Recovery in Small Children with Dilated Cardiomyopathy. Pediatr Cardiol 2017;38:902-8.

17. Japp AG, Gulati A, Cook SA, et al. The Diagnosis and Evaluation of Dilated Cardiomyopathy. J Am Coll Cardiol 2016;67:2996-3010.

18. Sautin YY, Nakagawa T, Zharikov S, et al. Adverse effects of the classic antioxidant uric acid in adipocytes: $\mathrm{NADPH}$ oxidase-mediated oxidative/nitrosative stress. Am J Physiol Cell Physiol 2007;293:C584-96.

19. Ito $T$, Suwa M. Echocardiographic tissue imaging evaluation of myocardial characteristics and function in cardiomyopathies. Heart Fail Rev 2021;26:813-28.

20. Johnson RJ, Kang DH, Feig D, et al. Is there a pathogenetic role for uric acid in hypertension and cardiovascular and renal disease? Hypertension 2003;41:1183-90.

21. Zuo T, Liu X, Jiang L, et al. Hyperuricemia and coronary heart disease mortality: a meta-analysis of prospective cohort studies. BMC Cardiovasc Disord 2016;16:207.

22. Orlando A, Cazzaniga E, Giussani M, et al. Hypertension 
in Children: Role of Obesity, Simple Carbohydrates, and Uric Acid. Front Public Health 2018;6:129.

23. Buzas R, Tautu OF, Dorobantu M, et al. Serum uric acid and arterial hypertension-Data from Sephar III survey. PLoS One 2018;13:e0199865.

24. Okazaki H, Shirakabe A, Kobayashi N, et al. The prognostic impact of uric acid in patients with severely decompensated acute heart failure. J Cardiol 2016;68:384-91.

25. Kim H, Shin HW, Son J, et al. Uric Acid as prognostic marker in advanced nonischemic dilated cardiomyopathy: comparison with $\mathrm{N}$-terminal pro B-type natriuretic peptide level. Congest Heart Fail 2010;16:153-8.

26. Mogensen UM, Køber L, Jhund PS, et al. Sacubitril/ valsartan reduces serum uric acid concentration, an independent predictor of adverse outcomes in PARADIGM-HF. Eur J Heart Fail 2018;20:514-22.

27. Misra D, Zhu Y, Zhang Y, et al. The independent impact of congestive heart failure status and diuretic use on serum

Cite this article as: Li TT, Li HY, Cheng J. Changes of serum uric acid and its clinical correlation in children with dilated cardiomyopathy. Transl Pediatr 2021;10(12):3211-3217. doi: $10.21037 / \mathrm{tp}-21-537$ uric acid among men with a high cardiovascular risk profile: a prospective longitudinal study. Semin Arthritis Rheum 2011;41:471-6.

28. Fang J, Alderman MH. Serum uric acid and cardiovascular mortality the NHANES I epidemiologic follow-up study, 1971-1992. National Health and Nutrition Examination Survey. JAMA 2000;283:2404-10.

29. Culleton BF, Larson MG, Kannel WB, et al. Serum uric acid and risk for cardiovascular disease and death: the Framingham Heart Study. Ann Intern Med 1999;131:7-13.

30. Borghi C, Rodriguez-Artalejo F, De Backer G, et al. Serum uric acid levels are associated with cardiovascular risk score: A post hoc analysis of the EURIKA study. Int J Cardiol 2018;253:167-73.

31. Krishnan E, Baker JF, Furst DE, et al. Gout and the risk of acute myocardial infarction. Arthritis Rheum 2006;54:2688-96.

(English Language Editor: B. Draper) 